\title{
Developing of prediction models for soil profile and its parameters using Artificial Neural Networks
}

\author{
Hussein Elarabi ${ }^{1}$, Nahed F. Taha ${ }^{2}$ \\ ${ }^{1}$ Geotechnical Department, Building and Road Research Institute, University of Khartoum, Sudan \\ ${ }^{2}$ Building and Road Research Institute, University of Khartoum, Sudan
}

\section{Email address:}

husseinelarabi@yahoo.com (H. Elarabi), nahedelyas@hotmail.com (N. F. Taha)

\section{To cite this article:}

Hussein Elarabi, Nahed F. Taha. Developing of Prediction Models for Soil Profile and Its Parameters Using Artificial Neural Networks. Science Research. Vol. 2, No. 3, 2014, pp. 43-48. doi: 10.11648/j.sr.20140203.13

\begin{abstract}
Artificial neural networks (ANNs) are a form of artificial intelligence (AI), which in their architecture attempt to simulate the biological structure of the human brain and nervous system. In this report, back-propagation neural networks are used to predict soil classification and soil parameters of Khartoum State. The study was based on the available data collected from specified areas in Khartoum, and then the results were compared with data brought from actual boreholes to check the ANN model validity. The results indicate that artificial neural networks are a promising method in predicting soil classification and soil parameters of Khartoum State.
\end{abstract}

Keywords: Artificial Neural Networks, Soil Profile, Soil Parameters, Khartoum, Sudan

\section{Introduction}

Before construction of any structure, subsoil layers, attributes and all questions concern to it have to be known, this knowledge requires drilling of number of boreholes to determine the soil profile of a given area which is costly and consumes a lot of time. In Addition to costly and consuming a lot of time in drilling number of boreholes, the engineering properties of soil exhibit varied and uncertain behavior due to the complex and imprecise physical processes associated with the information of these materials The technique is known as Artificial Neural Networks (ANNs) and is well suited to model complex problems where the relationship between the model variables is unknown ${ }^{(3)}$.

\section{Development of Neural Network}

\subsection{Study Area}

The study area is located in Khartoum state about 363 $\mathrm{km} 2$.It lie between 442802.15 to $464546.8 \mathrm{~m}$ E latitude and $1708997.85 \mathrm{~m}$ to $1729803.866 \mathrm{~m} \mathrm{~N}$ longitude.

\subsection{Computer Program}

The computer software program 'Neuroshell2 version4' is used in this work to simulate the artificial neural network operation.

\subsection{Model Database}

The database includes more than 310 borehole logs of 108 sites spread over zone 3 in the center and west and east of Khartoum. In order to represent the respective locations of the data, a digital map of Khartoum city was used.

\subsection{Model Development}

Based on the comparisons between previously used methods, it can be concluded that a unified method should follow the following points:

- $\quad$ Start selection of architecture of network by ward nets with two hidden layers and default program for learning rate is 0.1 , momentum is 0.1 and initial weight is 0.3 , default of hidden neurons. After that change it to other architecture and parameters and compare the results till reach to optimal models (The process of selecting the optimal factors with the trial and error method)

- Decreasing the training time by following early stopping method for improving generalizing and avoid overfit to data. It is recommended in Neuroshell 2 that you train until the events since the minimum error factor is greater than 20,000 to 40,000 events (higher for recurrent nets). Don't set any other stopping criteria. 
- Decreasing the amount of training and testing data sets by take one borehole from any site, in the final compare the results which obtained from taking all boreholes with it, as named case $1 \&$ case 2 respectively.

- it can summarized that a unified method is driven basically from method 4 , it will take the same models in classifier and parameters in method 4,but it will add two models in parameters namely SPT model and Sieve model .Considering the points which mentioned above at applying the network for these models trial to improve the network.

- Filter classification models by excluded case two outputs have value 0 , providing not to be applied unless following soil group arrangement (UCSC), until reach to final classification predicted (UCSC). After training all values of predicted networks are entered in an excel sheet and the final predicted group sample is obtained by Figure (1).

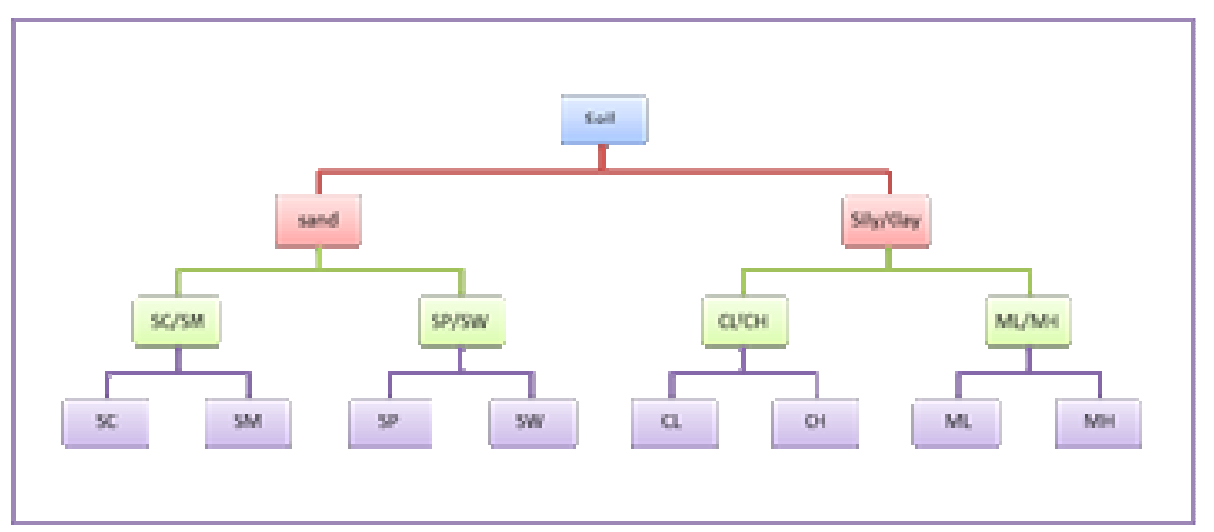

Fig.1. soil groups arrangement (UCSC)

- For this a unified method, it is decided to select the number of models is seven classification models, which enough to classify of soil according to (UCSC).Table (1) shows theirs names and input and output of six parameter models which are combination of all parameter models in previous methods (six models).Table (2) shows the parameter models and theirs names, input and output.

Table 1. Classifier models network" model 1 to model 7"

\begin{tabular}{lll}
\hline Model's Name & Input parameters & Output parameters \\
\hline Sand $\backslash$ clay silt model(1) & - E,N coordinates & Sand \\
& Claylsilt & Fine sand \\
Sand model(2) & - Descending depth from the altitude & Grade of sand \\
Fine sand model(3) & - Descending depth from the altitude & SC \\
& - E,N coordinates & SM \\
grade of sand model(4) & - Descending depth from the altitude & SP \\
& - E,N coordinates & Clayey layers \\
Clay $\backslash$ silt model(5) & - Descending depth from the altitude & Silty layers \\
& - E,N coordinates & CL \\
Clay plasticity model(6) & - Descending depth from the altitude & CH \\
Silt plasticity model(7) & - E,N coordinates & ML \\
\hline
\end{tabular}

Table 2. Parameter models network" model 8 to model 3"

\begin{tabular}{|c|c|c|}
\hline Model's Name & Input parameters & Output parameters \\
\hline Density model(8) & $\begin{array}{l}\text { - E,N coordinates } \\
\text { - Descending depth from the altitude }\end{array}$ & D.D(KN\m3) \\
\hline G.W.L model(9) & - E,N coordinates & W.D(m) \\
\hline Atterberg model(10) & $\begin{array}{l}\text { - E,N coordinates } \\
\text { - Descending depth from the altitude }\end{array}$ & $\begin{array}{l}\text { L.L }(\%) \\
\text { P.I }(\%)\end{array}$ \\
\hline Shear model(11) & $\begin{array}{l}\text { - E,N coordinates } \\
\text { - Descending depth from the altitude }\end{array}$ & $\begin{array}{l}\mathrm{C}\left(\mathrm{KN} \backslash \mathrm{m}^{2}\right) \\
\varnothing(\text { degree }\end{array}$ \\
\hline SPT model(12) & $\begin{array}{l}\text { - E,N coordinates } \\
\text { - Descending depth from the altitude }\end{array}$ & $\mathrm{N}$ value \\
\hline Sieve model(13) & $\begin{array}{l}\text { - E,N coordinates } \\
\text { - Descending depth from the altitude }\end{array}$ & Sieve \#200 \\
\hline
\end{tabular}




\subsubsection{Data Division}

These data sets are divided into two groups:

- 82 sites used as actual input data (training and testing data), where $80 \%$ from all data to training and $20 \%$ for testing data

- 26 sites used as rest data (valid data (production data), Figure (6.9) shows the location of these sites.

\subsubsection{Model Validation}

Once the training phase of the model has been successfully accomplished, the performance of the trained model has to be validated for an independent data set.

One of the most important criteria that has to be considered when assessing model performance is that good performance during training can always be attained. However, it is also important for the model to perform well for a set of data previously unseen by the model. Consequently, it is essential to check that the model performs consistently on all three data sets (i.e. training, testing and validation). The coefficient of correlation, $r$, the root mean square error, RMSE, and the mean absolute error,
MAE, are the major criteria that are used to evaluate the performance of the model $^{(2)}$.

\section{Results and Discussion}

\subsection{Classification Models}

Generally in this work our estimation for classification models performance depends on $\mathrm{R}^{2}$ (training and testing data) and percentage of success of all three data sets (i.e. training, testing and validation).

Also value of criteria $\left(R^{2}\right)$ and percentage of success in training and testing data showing Training efficiency of network models, while value of $\mathrm{R}^{2}$ and percentage of success in validation data was shown ability of network to predict soil profile, Table (3) Neural network results of classification models (case 1\&2). Fig (2) explains Training efficiency of network models while Fig (3) explains the classification models performance of validation data.

The percentage of success obtained after compared the actual soil layers type for each case study with predicted soil layers achieved by ANN.

Table 3. Neural network results of classification models (case 1\&2)

\begin{tabular}{|c|c|c|c|c|c|c|c|c|c|}
\hline No.Case & Type of Data & Criteria & Model 1 & Model 2 & Model 3 & Model 4 & Model 5 & Model 6 & Model 7 \\
\hline \multirow{6}{*}{ Case 1} & \multirow{6}{*}{ Training and testing } & $\mathrm{R}^{2}$ & 0.46 & 0.49 & 0.50 & 0.51 & 0.43 & 0.48 & 0.34 \\
\hline & & $r^{2}$ & 0.46 & 0.49 & 0.50 & 0.51 & 0.43 & 0.48 & 0.34 \\
\hline & & mse & 0.13 & 0.09 & 0.12 & 0.07 & 0.14 & 0.13 & 0.07 \\
\hline & & mae & 0.26 & 0.17 & 0.23 & 0.13 & 0.26 & 0.24 & 0.16 \\
\hline & & $\mathrm{r}$ & 0.68 & 0.70 & 0.71 & 0.72 & 0.65 & 0.70 & 0.59 \\
\hline & & $\%$ success & 80.6 & 90.3 & 83.4 & 90.7 & 81.9 & 81.6 & 90.1 \\
\hline \multirow{7}{*}{ case 2} & \multirow{6}{*}{$\begin{array}{l}\text { Training and testing } \\
\text { data }\end{array}$} & $\mathrm{R}^{2}$ & 0.38 & 0.21 & 0.71 & 0.64 & 0.45 & 0.62 & 0.76 \\
\hline & & $\mathrm{r}^{2}$ & 0.38 & 0.21 & 0.72 & 0.64 & 0.45 & 0.62 & 0.79 \\
\hline & & mse & 0.16 & 0.13 & 0.06 & 0.04 & 0.13 & 0.10 & 0.03 \\
\hline & & mae & 0.30 & 0.27 & 0.15 & 0.09 & 0.26 & 0.20 & 0.07 \\
\hline & & $\mathrm{r}$ & 0.62 & 0.46 & 0.85 & 0.80 & 0.67 & 0.79 & 0.89 \\
\hline & & $\%$ success & 78.0 & 83.1 & 90.8 & 94.0 & 81.6 & 86.8 & 97.1 \\
\hline & Production data & $\%$ success & 70.8 & 73.2 & 74.4 & 55.2 & 63.5 & 53.6 & 86.6 \\
\hline
\end{tabular}

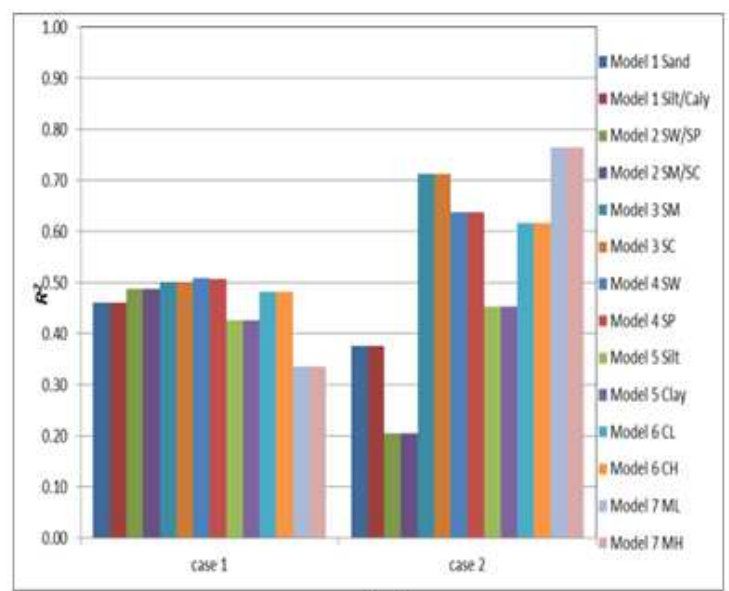

(a)

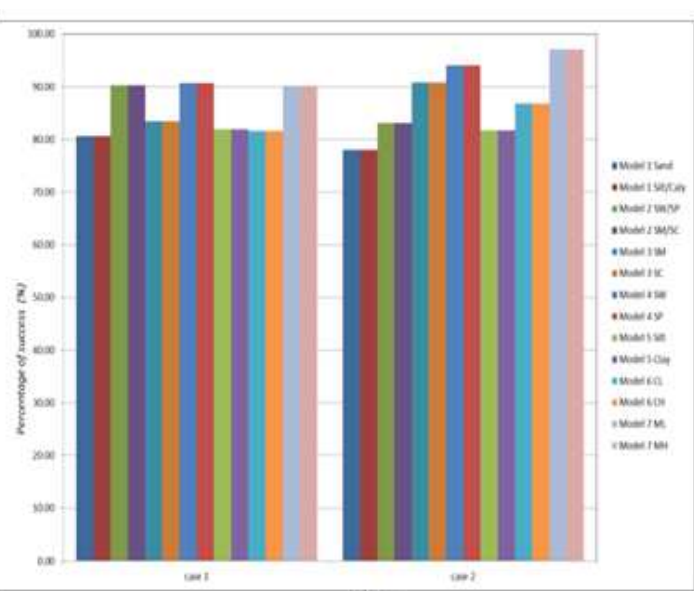

(b)

Fig.2. Training efficiency of network models of two cases 


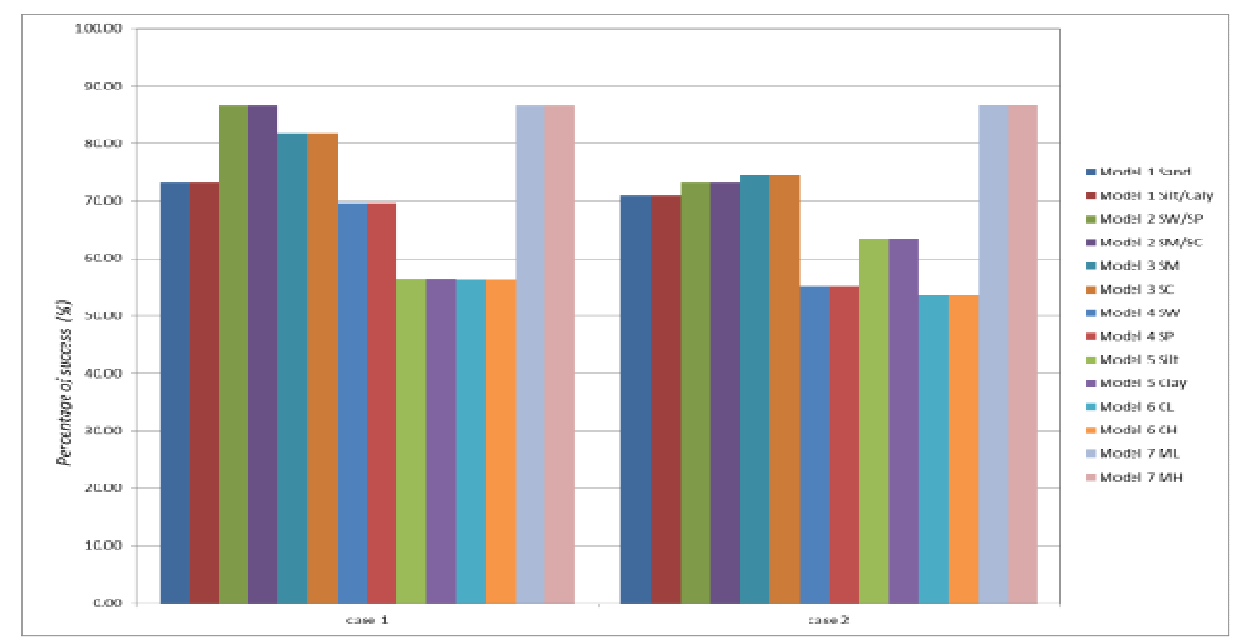

Fig. 3. The ability of network to predict soil profile (case 1\&2)

Table 4. Neural network results of parameter models (case 1\&2)

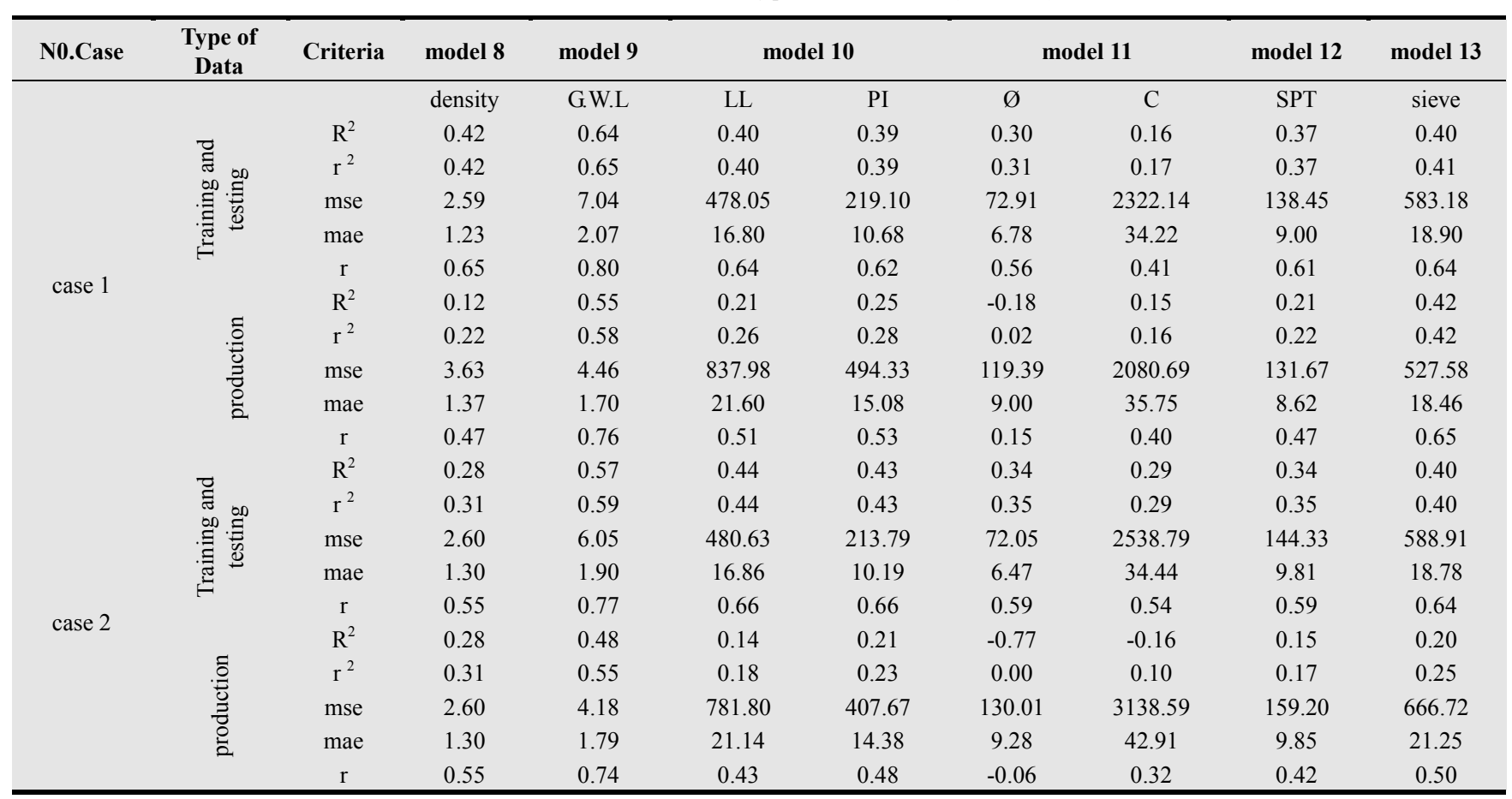

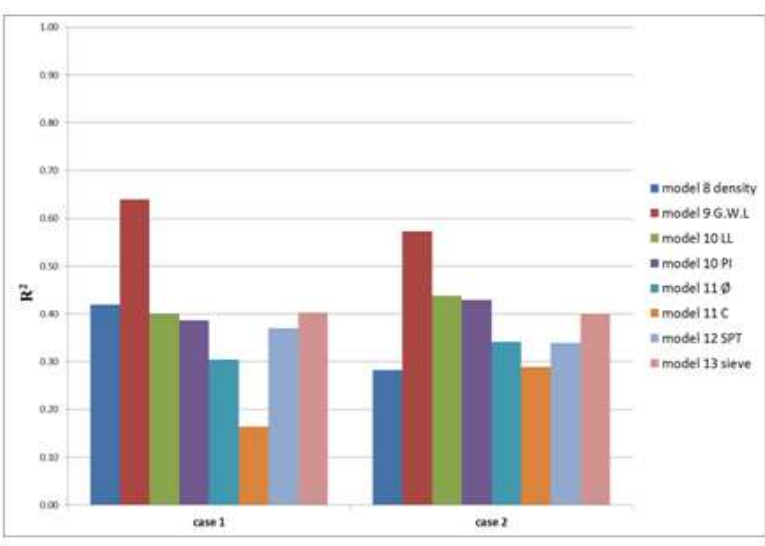

(a)

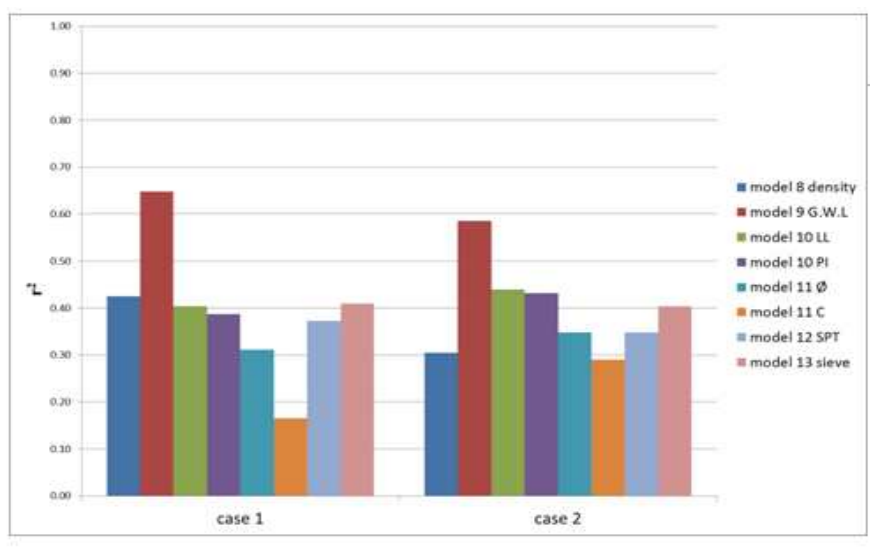

(b)

Fig. 4. Training efficiency of network models depend on value $R 2$ and $r 2$ in training and testing data 


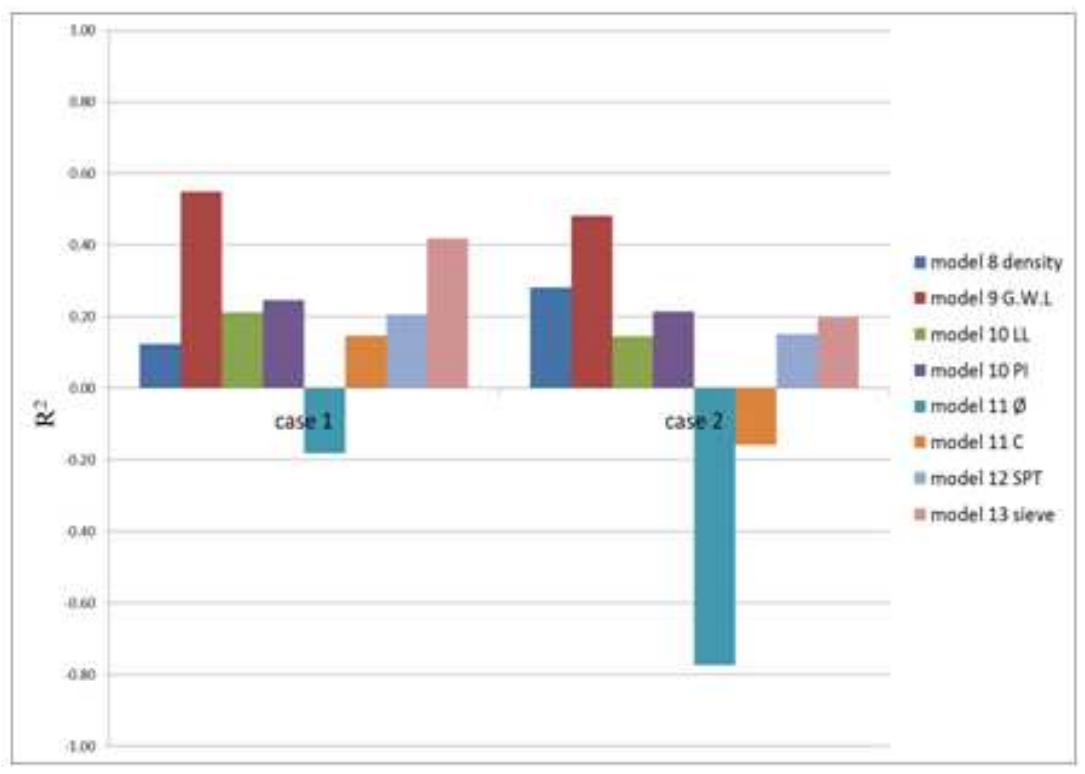

(a)

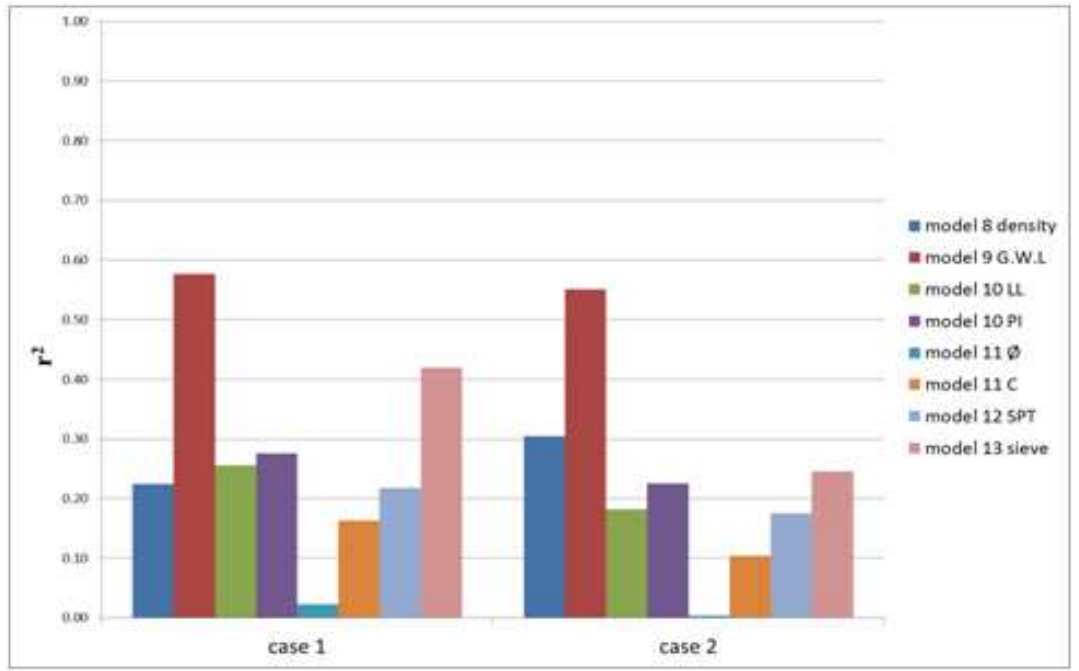

(b)

Fig. 5. The ability of network to predict parameters of soil depend on value $R^{2}$ and $r^{2}$ in valid data

Also values of criteria $\left(\mathrm{R}^{2}\right)$ and $\left(\mathrm{r}^{2}\right)$ in training and testing data show training efficiency of network models, while that values in validation data was shown ability of network to predict soil profile. Table (4) Neural network results of parameter models (case $1 \& 2$ ). Fig (4) explains Training efficiency of network models while Fig (5) explains the classification models performance of validation data. From these models of two cases and the figures we reach to:

- The results of models case 1 and models case 2 is closely

- The average $\mathrm{R}^{2}$ values are near to unity in all models in case $1 \& 2$; this is so good value for estimation, so the model demonstrates some degree of success.

- The percentages of success for classification models are high in all models, however with different values of $\mathrm{R}^{2}$ in both cases but the percentage is close.
- The results shows the ability of models to predict in both cases are good except the shear model

- $\quad$ The few entered data of c \& $\varnothing$ values in this model lead to considerable difference between the actual and predicted. Minus value of $\mathrm{r}^{2}$ were appeared which means that the result of case 2 worse than case 1 .

\section{Conclusion}

- The advantages of using neural networks to predict soil profiles is that neural networks are able to automatically create an internal distributed model of the problem during training, that make them a powerful and practical tool for soil classification prediction.

- Results obtained from the models show that ANNs with proper training is a good tool in prediction 
- Results obtained from the ANNs shear parameters model show that ANNs with proper training gives acceptable result especially for $\mathrm{C}$ parameter.

- ANNs are efficient tools when used as pattern classifier more than used for parameters prediction.

- Decreasing training time leads to reliable results especially in parameter models.

- In classification models, excluding the patterns which include the two outputs of value $(0)$ in both training and testing data will improve the results.

- The most models are not affect greatly by the amount of data in one site, data of all boreholes(case 1) may give better result than data of one borehole (case 2) except the case of shear model.

\section{References}

[1] El Hassan, M., (2009), "Prediction of Blue Nile Soil Profile Using Artificial Neural Network", M. Sc. thesis BBRI, University of Khartoum, Khartoum, Sudan.
[2] Mohammed, S. Elnasr (2009), "APPLICATION OF ARTIFICIAL NEURAL NETWORKS IN PREDICTION OF SOIL PROFILE IN SUDAN, MSc thesis BBRI, University of Khartoum, Khartoum, Sudan.

[3] Mohamed A. Shahin1; Holger R. Maier2; and Mark B. Jaksa3, (2002), "Predicting Settlement of Shallow Foundations using Neural Networks", Pp: (785-793).

[4] Mohamed, K.M. (2005), "Artificial Intelligence Applications in Geotechnical Engineering in Sudan”, MSc thesis, BBRI, University of Khartoum, Khartoum, Sudan.

[5] Nour Alfadul, Y.M. (2007), "Soil Profile Prediction Using Artificial Neural Networks in Sudan", MSc thesis BBRI, University of Khartoum, Khartoum, Sudan.

[6] Shahin, M. A., Jaksa, M. B., and Maier, H. R. (2001). "Artificial neural network applications in geotechnical engineering." Australia Geomechanics, 36(1), 49-62.

[7] M. A .Shahin, H. R. Maier \&Jaksa (2000), "Evolutionary data division methods for developing artificial neural network models in geotechnical engineering" Journal of Geotechnical Engineering - ASCE, Vol.1. 